\title{
Food cravings in UK and South Asian women with polycystic ovary syndrome
}

\author{
S.S Mulekar and Y. Jeanes \\ Health Sciences Research Centre, University of Roehampton, London, UK
}

Polycystic ovary syndrome (PCOS) is common endocrine condition characterised by a heterogeneous presentation of hyperandrogenism and ovulatory dysfunction. Weight management is the primary therapy in overweight/obese women with PCOS ${ }^{(1)}$. Food cravings has been identified as a significant partial mediator in the relationships between addictive-like eating, elevated body mass index (BMI) and binge eating episodes ${ }^{(2)}$ and ethnic variation in food cravings has recently been reported in non PCOS adolescents ${ }^{(3)}$, There is a paucity of studies exploring food cravings in women with PCOS. This study aims to observe food cravings in UK and South Asian women with polycystic ovary syndrome.

South Asian women with PCOS visiting a gynaecologist in Mumbai were recruited and completed the Food Craving Questionnaire-Trait (FCQ-T) ${ }^{(4)}$. UK participants completed a web-based survey, which included the FCQ-T. Ethical approval was granted by the process of the University of Roehampton Ethic Committee.

South Asian $(n=48)$ and UK women $(n=63)$ with PCOS were matched for self-reported BMI $(29 \cdot 1(5 \cdot 8)$ and 30.0 (6.1); NS) and age $(32.7 \pm 5.9$ and $32 \cdot 3 \pm 5 \cdot 7$; NS). South Asian women with PCOS reported significantly lower food craving scores compared with the UK women with PCOS (Table 1.).

Table 1. Food craving scores reported by women with PCOS.

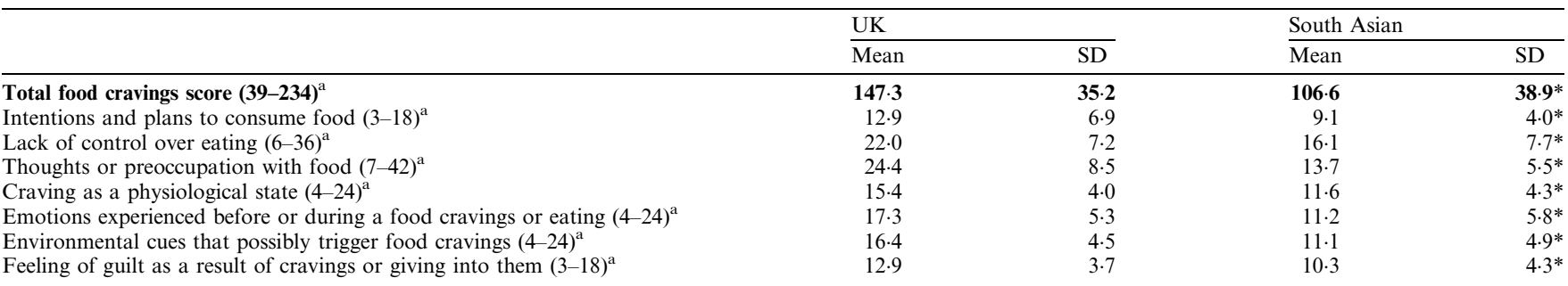

* significant difference between UK and South Asian participants, independent ttest $(\mathrm{p}<0 \cdot 01)$. ${ }^{\mathrm{a}}$ Possible ranges for the scores.

This is the first study to report food cravings in South Asian women with PCOS. South Asian women with PCOS reported lower food craving scores compared with UK women with PCOS and suggests future studies should explore cultural differences in food cravings.

1. Royal College of Obstetricians and Gynaecologists. (2014) Long-term consequences of polycystic ovary syndrome. Green-top Guideline No. 33.

2. Joyner MA, Gearhardt AN, White MA. (2015). Eating Behav. 19, 98-101.

3. Borgogna N, Lockhart G, Grenard JL, et al. (2015). J Acad Nutr Diet. 115, 759-66.

4. Cepeda-Benito A, Gleaves DH, Williams TL et al. (2000) Behav Ther 31, 151-173. 